\title{
Electrocardiogram Planned Start of Assessment Interval
}

National Cancer Institute

\section{Source}

National Cancer Institute. Electrocardiogram Planned Start of Assessment Interval. NCI

Thesaurus. Code C124012.

The period of time that the electrocardiogram assessment, done over an interval of time, is intended to begin. 